\title{
EVOLUTION OF THE ACTION-BASED APPROACH AND THE ADJUSTMENT OF COLLABORTIVE PRACTICES FOR PROFESSIONAL PURPOSES: THE CASE OF THE LINGUISTIC UNIVERSITY OF NIZHNY NOVGOROD
}

\author{
[EVOLUTION DE L'APPROCHE ACTIONNELLE ET \\ ADJUSTEMENT DES PRATIQUES PROFESSIONNELLES: LE CAS \\ DE L'UNIVERSITÉ LINGUISTIQUE DE NIJNI NOVGOROD]
}

\author{
Elena Porshneva - Indira Abdulmianova
}

\section{doi: 10.18355/PG.2017.6.1.13}

\begin{abstract}
Language is a social product and an instrument of its constitution. This idea is productive for contemporary language-culture didactology. This contribution aims to demonstrate the steps that this integrative pedagogical science has taken to realize and learn to exploit it. The authors report their experience in adjusting the professional approach to the linguistic training of future translators / interpreters during collaborative Tandem learning organized at the Faculty of Translators and Interpreters of the Nizhny Novgorod Language University (Russia).
\end{abstract}

\section{Key words}

collaborative learning, training of translators/interpreters, language practice, Tandem, action-oriented approach, contextual language training

\section{Résumé}

La langue est un produit social et un des instruments de sa constitution. Cette idée s'avère productive pour la didactologie des langues-cultures contemporaine. La présente contribution vise à démontrer les étapes que cette science pédagogique intégrative a franchies pour en prendre conscience et apprendre à l'exploiter. Les auteurs font part de leur expérience d'ajuster l'approche actionnelle aux finalités professionnelles de la formation linguistique de futurs traducteurs / interprètes lors de l'apprentissage collaboratif en Tandem organisé à la faculté de traduction et interprétation de l'Université linguistique de Nijni Novgorod (Russie).

\section{Mots clés}

apprentissage collaboratif, formation de traducteurs/interprètes, pratique langagière, Tandem, approche actionnelle, formation linguistique contextuelle

\section{Introduction}

Nous vivons dans une société ouverte aux autres cultures où la collaboration interculturelle en présentiel et à distance devient incontournable. Notre conception de la formation linguistique universitaire est en train de changer : dans une société cognitive on apprend autrement. La didactique des langues s'oriente vers un enseignement plus naturel, plus personnalisé et plus créatif, centré sur le développement de la personnalité de l'apprenant, de son identité langagière, de ses compétences, tirant profit des interactions avec l'enseignant et d'autres apprenants. Ce qui entraîne un changement profond dans les formes d'apprentissage selon les tâches et les situations réelles de communication. Les pédagogies de tâches et de projets enrichissent leur éventail non seulement par des activités ludiques et simulations 
interculturelles, mais aussi par des projets d'interaction et pratiques langagières collaboratives d'enseignement.

Le point de départ de cette réflexion sur l'enseignement du FLE dans l'optique du traducteur repose sur le constat que les dernières technologies de pointe et les pratiques d'enseignement en tandem deviennent indispensables vu leur importance dans le développement et enrichissement les interactions sociales qui favorisent l'autonomie et la responsabilisation des apprenants.

Cet article a pour but de montrer l'efficacité didactique de l'intégration des pratiques de Tandem dans l'enseignement du FLE à la faculté de traduction et d'interprétation.

\section{De la méthodologie traditionnelle d'enseignement des langues vers l'approche actionnelle dans le contexte professionnel}

La méthodologie traditionnelle d'enseignement des langues vivantes appelée "grammaire-traduction", vise à former de bons lecteurs de "grands textes littéraires". A partir des années 60-70 elle cède peu à peu sa place à la méthodologie communicative qui se construit en Europe élargie suite au développement des sciences portant sur des problèmes de l'identité langagière, de ses activités langagières, cognitives et socioculturelles, (sociolinguistique, psycholinguistique, psychologie cognitiviste, culturologie, anthropologie culturelle etc). Chaque discipline fait un apport pour la formation de la didactique des langues ou "la didactologie des langues-cultures" d'après R.Galisson. (Galisson.1986) La nouvelle science pédagogique intégrative, détachée de la linguistique appliquée, a pour objet l'étude des conditions et des modalités d'enseignement et d'appropriation des langues et cultures en milieu non naturel. Son objet ainsi défini, l'enseignement est conçu comme le guidage des apprenants dans leur acte d'appropriation linguistico-culturel. La découverte par des didacticiens et psychologues occidentaux des recherches des savants soviétiques, de leurs théories, notamment de la théorie psychologique de l'activité de A.N. Leontiev, de la théorie culturelle et historique de L. Vygotsky, de la conception dialogique de la culture de M.M. Bakhtine, de la théorie psychologique de l'activité langagière de A.A. Leontiev a façonné la didactique des langues en étoffant le champs d'analyses interdisciplinaires, ouvrant de nouveaux aspects de recherches scientifiques autour des notions-clés «activité», « acte de parole », " communication », « compétence communicative», «identité langagière», «discours», « typologie et genres de discours ", " opérations cognitives ", "stratégies cognitives», " l'interculturel » etc. La science se développe activement et englobe de nouveaux domaines de la connaissance.

Selon D.Abry, " l'approche communicative a constitué une véritable révolution » (Abry. 2007). En effet, c'est à partir de cette époque qu'on commence à dissocier et à traiter à part les quatre compétences langagières (compréhension orale et écrite, expression écrite et orale). L'enseignement des langues prend en compte la typologie des textes et la variété des discours, définit la structure interne des compétences communicative, linguistique, discursive, sociolinguistique, socioculturelle, stratégique et pragmatique. La mise en oeuvre des supports authentiques contextualise le processus d'apprentissage, crée le milieu réel d'acquisition d'une langue. L'approche communicative répondait à tous les niveaux aux soucis et besoins du Conseil de l'Europe qui encourage toutes les tentatives d'élaborer «les méthodes d'enseignement et d'apprentissage qui aident les jeunes, mais aussi les moins jeunes, à se forger les savoirs, savoir-faire et attitudes dont ils ont besoin pour acquérir davantage d'indépendance dans la réflexion et dans l'action afin de se montrer plus responsables et coopératifs dans leurs relations à autrui. En ce sens, ce travail contribue à promouvoir une citoyenneté démocratique " (CECR, 2001). Toutes ces idées aussi bien que les fondements théoriques et pratiques de la méthodologie communicative 
sont fixées dans l'ouvrage clé pour la didactique des langues « le Cadre européen commun de référence pour l'apprentissage et enseignement des langues ».

Comme le souligne Ch.Puren, c'est le passage de l'universalité vers la spécificité les langues et cultures. La méthodologie "grammaire-traduction" se concentrait sur la traduction (thème et version) en tant que moyen efficace de préparer les apprenants à lire en langue étrangère. L'objectif de la méthodologie était d'apprendre aux élèves à retrouver dans les grands textes classiques le «fonds commun de toute l'Humanité » les valeurs universelles.

L'approche communicative oriente les apprenants vers la recherche des particularités dans la langue-culture cible, vers les spécificités des cultures étrangères : à partir du moment où l'on accède à des documents plus nombreux, plus variés et plus récents, on perçoit les cultures comme vivantes et différentes les unes des autres.

Adressons-nous à la publication de Ch. Puren, parue en 2002 consacrée à la revue des perspectives actionnelles et culturelles en didactique des langues-cultures (Puren, 2002).

L'auteur développe l'idée que les différentes méthodologies élaborées depuis des années $60 \mathrm{du}$ siècle précédent apportent leurs contributions à l'enrichissement de la didactique des langues /didactologie des langues-cultures. Construites sur un principe d'adéquation entre ce qu'on demande aux apprenants de réaliser en langue étrangère (perspectives actionnelles) et le nouvel objectif social de référence (perspectives culturelles) ces méthodologies cherchent à répondre aux besoins de la société en mobilisant toutes les ressources pour complémenter l'approche qui est en vigueur. Citant à titre d'exemple le cas du Cadre européen commun qui fait suite à l'approche communicative et dont trois versions apparaissent entre 1996 et 2001, Ch. Puren décrit l'évolution historique des formes d'adéquation entre perspectives actionnelles et perspectives culturelles et montre comment un dépassement de la perspective actionnelle ou culturelle amène à élaborer et à mettre en œuvre une culture d'action commune dans le sens d'un ensemble cohérent de conceptions partagées : c'est très précisément ce processus qui constitue l'objet et l'objectif de ce que l'auteur propose d'appeler la «perspective co-actionnelle et co-culturelle ». L'objectif de l'approche communicative se complexifie en mettant en valeur un nouvel aspect d'enseignement/apprentissage des langues apporté par les approches, élaborées sur les mêmes principes et mises en place au cours des dernières décennies (interculturelle, interactionnelle, cognitive et d'autres) qui enrichissent la méthodologie actionnelle. En caractérisant les avancées de la recherche en didactique des langues J.-P. Cuq et I. Gruca mettent en évidence la constitution d'une " méthodologie-compromis » qui «s'était installée sur les lignes de force du courant communicatif avec notamment un retour à l'enseignement plus systématique de la grammaire, du lexique et de la correction phonétique " dont la méthodologie communicative constitue la composante de base (Cuq, Gruca. 2003.249). Cette méthodologie englobant les dernières modifications portera le nom de "l'approche actionnelle ».

Développant le schéma de Ch.Puren on peut voir comment élargissent l'objet et les objectifs de la didactique des langues / didactologie des langues-cultures.

L'objet d'études de la méthodologie de l'enseignement des langues change. L'étude de la langue comme système linguistique (de ses aspects phonétique, lexical et grammatical) passe au deuxième plan, mettant au premier la langue en tant qu'instrument de la communication interculturelle. Plus tard, le rôle de la langue comme objet d'étude de la didactologie des langues-cultures se complexifie. Du point de vue didactique la langue est considérée aussi comme moyen du développement de la personnalité de l'apprenant, comme l'instrument de l'initiation à la culture de l'Autre, l'instrument de coopération et co-action interculturelle, outil de formation de l'identité langagière professionnelle, 
Examinons comment se transforment les objectifs de l'enseignement des langues:

La prédominance des savoirs déclaratifs sur les savoirs opérationnels, la reproduction des connaissances linguistiques systématisées cèdent leur place à la construction des savoirs, savoir-faire, à l'enrichissement de l'expérience communicative. Si la méthodologie traditionnelle visait à former un lecteur, apprenant à expliquer un texte littéraire, la méthodologie actionnelle vise à former un communicateur / interlocuteur en développant les compétences langagières et communicatives des apprenants.

En ce qui concerne les supports didactiques nous voyons que les didacticiens passent de grands textes littéraires à la littérature contemporaine, des textes didactiques, fabriqués à des fins d'enseignement, aux documents authentiques au format textuel, visuel, audiovisuel, scriptovisuel ou graphique.

Concernant l'aspect culturel de l'enseignement des langues citons encore une fois $\mathrm{Ch}$. Puren qui met en évidence l'opposition de la méthodologie traditionnelle orientée vers l'universalité (le fonds commun) des cultures et de la méthodologie communicative ouverte à la recherche des convergences et divergences dans les deux cultures (maternelle et étrangère) sensibilisant les apprenants aux spécificités de la culturecible. Ce qui est très significatif pour la didactique de nos jours c'est son intérêt au malentendu et l'incompréhension dans la communication interculturelle. Mais en premier lieu c'est son intérêt d'apprendre aux étudiants à identifier les particularités de leur propre culture découvertes par l'Autre, « de faire apparaître en quoi notre propre culture nous est opaque, de sorte que nous fassions l'apprentissage de notre étrangeté » (Teyssèdre, 1984, cit par Abdallah-Pretceille,1995, p.211).

La révolution communicative touche aussiles si tuations de l'apprentissage. La méthodologie a suivi les stades suivants: celui de la situation non naturelle, hors contexte culturel et communicatif, celui de la réalité fictive pour aboutir à celui de la situation de communication interculturelle simulée. Les nouvelles technologies créent les conditions permettant d'effectuer l'appropriation d'une langue étrangère dans des situations réelles d'échange langagier, d'interaction et co-action interculturelles.

Les exercices offerts par la méthodologie traditionnelle et la didactologie des languescultures ne diffèrent que par leurs buts et leur rôle dans la formation d'automatismes et de savoir-faire. La méthodologie traditionnelle donnait la priorité aux exercices linguistiques d'entraînement en compréhension écrite (traduire littéralement / thème et version), concentrant l'attention des apprenants sur la forme : ceux de répétition, de transformation, de substitution, de réemploi. L'approche communicative a privilégié des activités ciblés sur le développement des compétences/performances, se focalisant sur le sens de l'énoncé : donner une explication, reformuler une idée, paraphraser, analyser, comparer, interpréter le fait, identifier la spécificité d'un document, reconstituer un texte. L'essentiel est que l'enseignement/apprentissage des langues prend en considération le développement des opérations cognitives nécessaires pour révéler l'information, décoder et comprendre le sens.

A côté des activités linguistiques demandant l'analyse et application des connaissances acquises, l'étude des documents, l'explication du texte, l'approche communicative offre des activités langagières, ciblées à effectuer des tâches communicatives, jeux de rôle, analyse discursive, simulations globales. Les dernières décennies, les recherches dans le domaine d'interactions verbales poussent la didactique des langues à intégrer la pédagogie de tâche et des projets qui stimule les pratiques collaboratives, co-actives et co-culturelles, dans lesquelles les apprenants mènent à bien leur projet commun qui est à la fois d'apprentissage et d'enseignement et où la langue reprend ses fonctions communicative et interactionnelle.

Les formes d'apprentissage subissent également une transformation considérable: formalisé dans la méthodologie traditionnelle, dans l'approche actionnelle le processus d'enseignement / apprentissage est centré sur la personnalité de l'apprenant, sur ses pratiques langagières collaboratives.

Slavonic Pedagogical Studies Journal, ISSN 1339-8660, Volume 6 Issue 1, February 2017 
Il apparaît clairement que l'approche actionnelle a complètement changé la méthodologie des langues étrangères en se focalisant sur l'acquisition de la compétence de communication, en précisant sa structure; en établissant les niveaux communs de référence, en découpant la compétence communicative en activités de communication langagière, en intégrant la notion de "tâche", de "besoins langagiers", de "pratiques langagières".

Comme nous voyons, la didactique des langues-cultures se développe vers l'authenticité du milieu d'apprentissage, vers l'éclectisme méthodologique, c'est à dire, la combinaison des éléments de différentes approches et différents outils pédagogiques en se focalisant sur le développement de l'identité langagière de l'apprenant sur la construction des savoirs procéduraux.

L'idée de la nécessité de concrétiser le milieu et le contexte pour l'enseignement/apprentissage des langues a ajouté un nouvel aspect à la didactologie des langues-cultures. La théorie socio-historico-culturelle du développement des fonctions mentales supérieures de L. Vygotsky a servi du point de départ pour l'élaboration de l'approche socio-constructiviste qui a renforcé et actualisé l'approche actionnelle. L'exploitation du rôle formatif des langues dans le développement des capacités professionnelles et intellectuelles constitue un des enjeux primordiaux du paradigme éducatif et formatif du XXIe siècle.

En Russie, la conception de L. Vigotsky et la théorie psychologique d'activité de A.N. Léontiev sont devenues la base pour l'approche dite « contextuelle », qui consiste en mise en contexte professionnel de l'enseignement supérieur (Verbitski, 1991) et l'enseignement des langues, en particulier. L'idée de contextualiser professionnellement l'enseignement/apprentissage des langues, de viser à enseigner la langue en tant qu'outil de formation professionnelle, a été approuvée et développée par les didacticiens de l'Université linguistique de Nijni Novgorod. L'approche contextuelle permet d'optimiser l'acquisition de la langue étrangère en poussant les étudiants à se sensibiliser au système de pensée et de communication professionnelle, à exploiter le potentiel de l'intelligence professionnelle. Le milieu d'apprentissage professionnellement contextualisé oriente les étudiants vers la construction et développement des compétences professionnellement significatives ainsi que des dispositions psychologiques, que le futur spécialiste devra mettre en œuvre en exerçant son métier.

\section{Spécificité de la formation linguistique dans l'optique du traducteur}

La traduction constitue une activité langagière spécifique, compliquée et polyfonctionnelle qui se base sur la maîtrise des quatre activités langagières (écoute, lecture, écriture, expression orale) dans deux langues, une performance spécifique de reexpression / reformulation et une performance particulière de médiation entre deux langues et deux cultures. L'exercice de cette activité demande de savoir mobiliser des connaissances extra-linguistiques (le bagage cognitif), d'avoir plusieurs capacités cognitives, psychologiques, sociales et culturelles pour effectuer la communication interculturelle et le passage d'une langue à l'autre. En outre, le traducteur doit posséder de très bonnes qualités rédactionnelles, une grande autonomie, un regard critique sur son travail pour être en mesure de s'auto-évaluer mais aussi une très bonne maîtrise des outils informatiques. L'interprète, quant à lui, doit être capable de traduire un discours oral ce qui nécessite en plus une agilité d'esprit, une grande souplesse verbale, une prononciation et une diction parfaites.

Suite à cette esquisse du portrait d'un traducteur/interprète, il apparaît que la formation linguistique de ce dernier doit être très complète, car le métier impose ses spécificités aux compétences langagières réceptives, reproductives et productives. Les activités professionnelles du traducteur/interprète sont liées surtout à la compréhension du sens du texte, au processus de formulation et reformulation de la 
pensée et à la recherche des équivalences interlinguistiques et interculturelles. Il est évident que la profession dans laquelle les langues accomplissent le rôle d'outil de travail demande une maitrise des langues professionnellement orientée. Les quatre compétences (expression écrite/orale, compréhension écrite/orale) demandent une attention spéciale, le lexique et la grammaire un intérêt particulier.

La traduction/interprétation est aussi un processus social appelé à assurer l'interaction sociale entre les représentants de deux cultures différentes et ne parlant pas une même langue. La méthode Tandem, construite sur l'idée de la socialisation de l'apprentissage d'une langue à travers des interactions peut aider à professionnaliser l'acquisition de la langue-cible et l'activation de la langue maternelle.

Ayant une longue expérience d'enseignement des langues aux futurs traducteursinterprètes nous pouvons constater que l'approche par compétences n'est pas toujours efficace pour rendre nos apprenants capables d'assurer l'interaction entre représentants de cultures différentes. Il nous a paru nécessaire d'ajuster notre méthode aux finalités de la formation spécifique de nos apprenants. Si on se pose l'objectif d'apprendre aux étudiants à construire des stratégies interactionnelles en milieu professionnel il s'avère nécessaire de s'adresser aux éléments des approches: communicative, active, cognitive, par compétences, contextuelle et interculturelle qui au total réalisent l'approche actionnelle.

La combinaison de ces approches intercomplémentaires et interdépendantes privilégie le travail sur le sens et permet de mettre en relief les détails des pratiques sociales en les rendant significatifs pour l'organisation de l'action langagière. En se complétant ils contribuent à la perception des cultures comme vivantes et différentes les unes des autres et mettent en valeur l'apprentissage comme un processus social qui repose principalement sur les interactions sociales. Ainsi, l'appropriation d'une langue étrangère devient-elle professionnellement significative pour la formation aux métiers des traducteurs / interprètes. D'emblée, l'attention des étudiants est concentrée sur les stratégies cognitives et les activités langagières de médiation, qui assurent l'interaction interculturelle. Parmi les pratiques les plus efficaces on distingue l'apprentissage en Tandem qui correspond le plus aux besoins et intérêt du métier du traducteur/interprète.

\section{L'apprentissage en Tandem à la faculté de traduction et d'interprétation de LUNN}

La faculté de traduction et d'interprétation à l'Université linguistique d'Etat à Nijni Novgorod célébrera son 55-ème anniversaire en 2017. De nos jours, la faculté constitue une vraie unité de formation et de recherche où s'effectue la formation en traduction et en interprétation, en journalisme international, aux études asiatiques chinoises et japonaises. Depuis la création de la faculté la gamme des langues s'est élargie considérablement : l'anglais, le français, l'allemand, l'espagnole, l'italien, le chinois, le japonais. On a des contacts avec des écoles de traduction et des facultés de traduction européennes. Les spécialistes en traduction participent à nos colloques, animent des ateliers, nos enseignants prennent part aux réunions internationales. Dans l'enseignement des langues et de la traduction règne l'approche par compétences.

\footnotetext{
Méthode Tandem et la formation de futurs traducteurs / interprètes

Depuis plusieurs années notre faculté accueille des étudiants étrangers participant au programme de formation inclusive. Les stagiaires ont le droit de fréquenter des cours de civilisation et littérature russe, de traduction, d'interprétation, de russe, etc. Nous les invitons aussi aux cours de langues étrangères lors desquels nous pratiquons la forme de l'apprentissage collaboratif appélée Tandem.
} 
«Tandem comme forme d'apprentissage collaboratif se réalise d'habitude entre deux personnes appartenant à deux communautés de langues maternelles différentes » (Lewis, Stickler.2007). Apparu vers les années 70 comme un des moyens d'apprendre une langue étrangère dans une situation de communication interculturelle, cette méthode permet d'organiser l'échange et le partage de son expérience communicative, de ses connaissances et stratégies de s'approprier la langue maternelle de l'Autre. En travaillant en Tandem, les participants combinent les rôles d'enseignant et d'apprenant, de commentateur et chercheur, interlocuteur et facilitateur, producteur et rédacteur du texte. Dans le cas du département d'études françaises, de traduction et d'intérprétation ce sont des étudiants russes et francophones (français, belges, suisses...) faisant leurs études aux facultés de traduction de leurs pays respectifs. Ce qui unit ces partenaires c'est ce qu'ils se forment aux métiers de traducteurs ou interprètes du français vers le russe et du russe vers le français.

Alors, cette forme d'apprentissage reçoit encore plus d'importance car elle devient un moyen supplémentaire de professionnalisation de leur formation linguistique. L'objectif de découvrir leurs cultures respectives doit être affiné par l'idée de savoir faire découvrir sa propre culture - une compétence cruciale pour les traducteurs/intreprètes car ils vont travailler à la frontière des deux cultures.

En prenant en compte les effets bénéfiques de la méthode Tandem pour notre public et certaines contraintes objectives, nous l'appliquons en présentiel. Cela nous permet d'augmenter son potentiel formatif en profitant au maximum de ses avantages - le face-à-face, l'entraide, la réactivité, l'échange, la spontanéité. Le Tandem en présentiel est très réactif. L'échange se faisant en temps réel, les partenaires n'ont pas beaucoup de temps pour réfléchir, pour consulter des dictionnaires - les participants sont contraints à mobiliser toutes leurs compétences (savoirs, savoir-faire, capacités, aptitudes, qualités, attitudes) pour résoudre des tâches communicatives.

Ce type de Tandem incite à utiliser les stratégies interactionnelles dans des conditions naturelles, demandées par le besoin personnel et non par le professeur. Les étudiants apprennent à solliciter de l'aide, à introduire une idée, à demander de reformuler, à appliquer des stratégies métacommunicatives (s'assurer qu'ils ont été bien compris, entamer son intervention, contrôler l'attention et la compréhension des interlocuteurs confirmer son intérêt etc.).

\section{Durée et fréquence}

Les résultats de notre expérience montrent que la méthode est plus efficace si l'apprentissage dure plus d'un semestre. La fréquence des cours Tandem dépend de la disponibilité des apprenants étrangers. Bien entendu, les meilleurs résultats étaient obtenus par les étudiants qui ont pu être présents à presque tous les cours de français prévus par le programme (12 heures par semaine au maximum) pendant une année d'études. Mais à notre grand regret cela arrive très rarement et le plus souvent les apprenants étrangers fréquentent 2 ou 3 séances par semaine.

\section{Cadre matériel}

Les cours se déroulent dans les salles équipées par un ordinateur, un tableau blanc, les apprenants et le professeurs s'installent autour d'une table ovale. Cela permet de créer les mêmes conditions pour tous les participants: le professeur n'est pas opposé au groupe, chaque apprenant est face-à-face à tout le monde, les apprenants francophones s'installent côte-à-côte ce qui leur donne plus de confort psychologique nécessaire au début (avec le temps on va former des sous-groupes interculturels pour accomplir une tâche mais il vaut mieux ne pas prévoir des activités pareilles avant la fin de la période de l'adaptation) les participants ne sont pas installés loin les uns des autres ce qui facilite l'entraide et collaboration.

\section{L'enseignant et son rôle en Tandem}

Il faut préciser que nous pratiquons une forme spécifique de Tandem - celui en présentiel avec l'enseignant car nous sommes persuadés qu'au début, les étudiants ont 
besoin de l'aide du professeur. Il leur apprend à travailler en collaboration interculturelle de façon qu'elle devienne propice pour leur autoformation. Le rôle de l'enseignant se modifie, il devient médiateur, provocateur, organisateur, communicateur, animateur. Il veille à la coordination didactique et procédurale des cours (sélection des sujets pertinents pour son public conformément à leur niveau et bagage cognitif, l'utilisation des deux langues de façon bénéfique pour tous les participants, etc.). C'est lui qui guide les étudiants dans la découverte de l'asymétrie des formes linguistiques de deux langues, des particularités comportementales de la culture maternelle révélées par les représentants de l'autre culture. Il anime le cours et peut arrêter l'échange à n'importe quel moment pour attirer l'attention à un détail qui risque passer inaperçu, et demander d'expliquer ou de reformuler la notion ou l'idée qui n'est pas comprise, ou comprise autrement. C'est lui qui évalue l'apport de chaque participant à l'échange et le rectifie si nécessaire. Peu à peu il minimise sa participation en donnant plus d'autonomie aux étudiants mais il ne le fait qu'après s'être assuré que ceux-là ont bien compris les règles du fonctionnement efficace du Tandem.

\section{Caractéristiques du public}

Effectif

Une autre spécificité de notre méthode est que les étudiants ne travaillent pas en pairs permanents. Nous n'organisons pas de cours supplémentaires pour l'apprentissage en Tandem et invitons les apprenants étrangers aux cours de français prévus par le programme académique de nos étudiants. Il y a deux ou trois locuteurs de français sur une dizaine de locuteurs de russe. Mais l'expérience fait preuve que cette structure peut être assez efficace à condition que le professeur sache l'organiser d'une manière appropriée. Il est à noter que nous tâchons d'éviter la présence de plus de deux cultures à un même cours et ne pas former des groupes avec les représentants de pays francophones différents, surtout au début, car cela complique le processus.

\section{Niveau}

Le plus souvent les étudiants francophones sont invités à participer aux cours de français de la deuxième année d'études. Cette étape est choisie par plusieurs raisons pédagogiques et didactiques.

La première année d'études vise la construction des bases linguistiques et débouche à un niveau A2. Ils sont en train de construire leur propre image de la culture-cible. D'autre part à cette étape les apprenants forment et développent leur aptitude de l'apprentissage collaboratif professionnel d'une langue étrangère. Cette capacité se construit pendant les cours de français lors du travail en groupes et sous-groupes. Cette expérience leur sera bien utile en Tandem.

L'étape suivante est centrée sur l'approfondissement des bases linguistiques et le développement des acquis pré-professionnels. Les apprenants sont déjà capables de formuler leurs idées sur des sujets encore élémentaires mais leur bagage linguistique est déjà suffisant pour entrer en interaction avec les apprenants étrangers. Les sujets abordés à cette étape dépassent le domaine personnel (savoir se présenter, parler de soi, de ses occupations etc) et prévoient les interactions sociales simples de tous les jours (aller au restaurant, au magasin, chez le médecin, ...). C'est ici qu'on aura besoin de l'aide des partenaires étrangers.

Les cours de langue en troisième et quatrième années ont pour but le développement des compétences textuelles, interculturelles et discursives et passent au deuxième plan, la priorité donnée aux cours de traduction et d'interprétation.

Expérience langagière

L'organisation du travail en Tandem se base aussi sur l'expérience langagière des participants. Nous dégageons les types suivants d'apprenants:

1. Apprenant russe qui n'a pas d'expérience de communication avec des étrangers. Il sera d'abord angoissé par la présence d'un étranger dans le milieu qui est le sien, il 
analysera l'apparence de ce nouvel agent et comparera ses premières impressions avec l'image qu'il s'est déjà fait des représentants de ce pays en se basant sur ses connaissances présupposées. Heureusement, le nombre d'étudiants de ce type diminue d'année en année.

2. Apprenant russe qui a une expérience de communication avec des étrangers mais elle ne se faisait jamais en français. Il se sentira plus à l'aise que l'apprenant du type précédent. Il a déjà un esprit critique envers son attitude et son image des étrangers et il est prêt à le modifier plus rapidement.

Ces deux catégories d'étudiants auront un défi: commencer à utiliser la langue qu'ils apprennent - parler français avec les natifs et le professeur doit les aider à faire ce défi sans se décevoir.

3. Apprenant russe qui a une expérience de communication avec des francophones ou non-francophones mais en français. Cette catégorie est la plus prête aux échanges et ils seront les premiers à mener les interactions, mais le professeur doit veiller à ce que les autres interviennent aussi.

4. Apprenant francophone qui a une expérience très limitée de communication en russe. Cette expérience peut être liée juste au voyage qu'il vient de faire. Il a aussi besoin de soutien du professeur qui doit l'encourager les premiers temps.

5. Apprenant francophone qui a une expérience bonne ou suffisante de communication en russe. Il sera aussi le premier à entrer en contact avec le groupe et donnera ainsi à son compatriote du temps pour s'adapter.

Le professeur doit tenir compte des particularités de ces catégories pour pouvoir rendre le travail plus naturel, plus confortable pour tout le monde.

\section{Premiers échanges - premières découvertes}

Nous voudrions que Tandem nous aide à engager les étudiants dans une diversité de tâches authentiques, personnelles et spontanées. En réalité pour certains étudiants ce type de communication devient stressant même dans la langue maternelle en présence des partenaires étrangers. Un des objectifs de Tandem est d'apprendre aux futurs traducteurs / interprètes à gérer le stress.

Les premiers échanges passent dans une ambiance un peu tendue. Les apprenants, francophones aussi bien que ceux russes, éprouvent de l'anxiété langagière inspirée par le partenaire de communication étranger et peuvent se replier sur eux-mêmes lorsqu'ils doivent prendre la parole devant tout le groupe. La tâche de l'enseignant est de faire parler les participants en créant des situations où les étudiants aient le désir de communiquer avec les locuteurs natifs. Cela demande une préparation particulière.

L'essentiel qu'il fasse connaissance avec les apprenants étrangers avant le cours pour se faire une idée de leur niveau de russe, de leur expérience langagière et culturelle en russe. Il précise depuis quand ils apprennent le russe, si c'est leur premier séjour en Russie, quels autres pays ils ont visités, etc. Cette information sera utile pour préparer le premier cours d'apprentissage en Tandem. Tous les apprenants sont priés de venir quelques minutes avant le cours pour s'adapter à une nouvelle situation. Le professeur observe les comportements des étudiants et choisit sa tactique.

Une fois le cours commencé le professeur annonce que dès lors certains cours de langue seront organisés autrement. Il présente la méthode Tandem en soulignant son potentiel pour la formation de futurs traducteurs / interprètes. Il salue les nouveaux membres du groupe en leur donnant d'emblée ce statut pour les faire integrer au groupe et invite tout le monde à se faire connaissance. Cette introduction est aussi nécessaire pour que tous les participants puissent prendre conscience des nouvelles conditions d'apprentissage.

Le professeur prévoit plusieurs activités qui permettront aux participants de se connaitre. Il peut demander les étudiants russophones de présenter l'un l'autre en français. Cette activité développe la capacité de transmettre une information 
personnalisée. Le choix de l'information à transmettre montre les relations qui relient les membres, les traits auxquels on fait attention. Il est important aussi qu'on exprime son attitude envers l'apprentissage. A l'étape suivante, ce sont les étudiants étrangers qui présentent l'un l'autre en russe. D'habitude, il se laissent guidés par les points mentionnés par les russophones en s'insérant ainsi dans la structure du groupe.

Si tous les apprenants francophones ont une expérience interculturelle et n'ont pas besoin du soutien de leur compatriote on peut proposer une autre activité pour se présenter. On peut diviser les étudiants en sous-groupes dans lesquels il y aura un étranger et donner quelques minutes pour qu'ils se présentent les uns aux autres. Ensuite, chaque sous-groupe présente ses membres à tout le groupe. Les langues doivent se succéder.

Ainsi dès la première activité en Tandem les apprenants accomplissent le rôle de médiateurs en utilisant la langue cible en guise d'instrument. Pour la plupart d'entre eux cette expérience sera la vraie première dans leur vie professionnelle.

Un bon signe de réussite du premier cours est que les étudiants partent ensemble après la séance. Cela signifie que le professeur a réussi à organiser une ambiance propice pour la formation du groupe Tandem et les étudiants, de leur côté, ont fait un effort nécessaire pour cela.

Pour prouver que la notion du groupe est importante dans notre cas citons le témoignage d'une stagiaire française :

Malgré toutes ses différences scolaires, intellectuelles, sociales, l'unité des groupes est souvent très forte. En effet, pour privilégier la communication orale, chaque groupe est constitué d'environ douze étudiants qui étudieront ensemble pendant cinq ans. Ces petits groupes qui passent de nombreuses heures ensemble à l'université, qui rencontrent les mêmes difficultés, apparaissent comme des petites familles où chacun s'entraide. Il peut par exemple paraître curieux à un étudiant français de fêter son anniversaire durant un cours à l'université avec son professeur et d'autres étudiants. Et pourtant, en Russie, c'est chose courante.

Certains vous diront que l'âme russe se caractérise par des rapports humains beaucoup plus profonds que dans la plupart des pays occidentaux. L'ambiance de la classe est donc généralement très amicale, chaleureuse. Une autre conséquence $d u$ nombre restreint d'étudiants dans chaque groupe concerne la relation que l'enseignant entretient avec ses apprenants. Ceux-ci ont en effet la chance d'avoir des rapports privilégiés avec leur professeur, la barrière institutionnelle qui existe entre le professeur et ses apprenants en Europe est presque inexistante en Russie. Ces " petites familles ", très maternées par leur professeur, rencontrent alors de graves problèmes d'autonomie. Cette particularité des étudiants russes est le résultat de toute une tradition scolaire qui laisse peu de place à l'initiative. Actuellement, ce manque d'autonomie et de responsabilités vis-à-vis de son propre apprentissage est un important désavantage pour les apprenants russes.

Ce témoignage montre bien les divergences entre les apprenants russes et ceux francophones dont le professeur doit tenir compte en prévoyant les activités pour intégrer les étrangers dans le groupe et stimuler les russes de prendre plus d'initiative et d'être plus autonome et responsable.

\section{Le déroulement des cours et exemples des tâches et des activités}

Les cours les plus adaptés à l'apprentissage collaboratif sont «la grammaire contrastive » et «l'interaction interculturelle».

\section{La grammaire contrastive}

La première question qu'il convient de se poser, c'est en quoi consiste l'apprentissage de la grammaire en Tandem. Normalement, l'apprentissage de la grammaire est vu 
comme processus de l'appropriation des formes et des structures morpho-syntaxiques d'une langue-cible que l'apprenant sera capable d'opérationnaliser dans son discours.

L'approche que nous avons choisie pour ce type de cours porte le nom de conscientisation grammaticale. Cette approche est orientée vers la formation chez l'apprenant des connaissances explicites, déclaratives - une sorte de connaissance intellectuelle sur un sujet quelconque. Nous ne pouvons pas dire que l'idée d'apprendre la grammaire d'une langue d'une manière consciente est nouvelle. Comme nous l'avons déjà signalé ci-dessus l'idée de l'enseignement explicite des phénomènes grammaticaux réapparaît dans les années 80 du XX-e siècle. Sa réapparition a été causée par le fait que les activités communicatives pratiquées en classe de langue n'étaient pas suffisantes pour favoriser un usage productif des aspects formellement marqués d'une langue étrangère. Les erreurs dues en partie à l'interférence de la langue maternelle et pour une autre partie à la surgénéralisation et à la simplification des règles d'une langue étudiée se fossilisaient et le niveau de la précision/ exactitude grammaticale atteint par la plupart des apprenants restait très bas. Alors, le moment est venu de s'interroger sur ce que la conscientisation grammaticale peut apporter pour améliorer cette situation.

L'utilisation de cette approche dans notre contexte d'apprentissage nous paraît justifiée pour trois raisons. Premièrement, parce qu'il s'agit des apprenants de deuxième année. Pour certains d'eux la comparaison explicite du système grammatical de la langue-cible avec celui de la langue-source constitue un moyen efficace de se construire assez rapidement une représentation mentale de ce système. Deuxièmement, parce qu'il s'agit des apprenants adultes dont le système cognitif atteint son point maximal pour ce qui concerne l'attention, la concentration, la mémoire, les aptitudes d'analyse ou de généralisation. Troisièmement, parce qu'il s'agit de la compétence grammaticale qui acquiert une valeur professionnelle dans la formation linguistique de base des traducteurs/interprètes.

Pour voir dans quelle mesure les connaissances grammaticales dans la langue maternelle influencent la formation de la compétence grammaticale en langue-cible nous avons prévu des activités de conscientisation au cours de l'apprentissage en Tandem.

Sur le plan pratique les cours comportent trois aspects :

1. discussion / la mise en question des modèles linguistiques traitant le problème du phénomène grammatical dans la perspective comparative,

2. activités de comparaison du fonctionnement du phénomène étudié dans les deux langues, ainsi que l'analyse des textes en russe et en français et discussion des textes actualisant les phénomènes étudiés au niveau de problèmes soulevés et d'idées évoquées,

3. la vérification des hypothèses sur les effets du sens produits par ce phénomène dans les deux langues.

En ce qui concerne la discussion de modèles grammaticaux, le travail consiste en adaptation et en présentation des modèles correspondants dans la langue maternelle. Les étudiants sont censés y découvrir des points communs et des différences et les présenter sous forme de schémas / tableaux. Ils doivent aussi découvrir si les catégories (notions / idées générales / concepts) figurant dans les modèles discutés font partie du système de la langue maternelle et dans quelles formes sont revêtus ces concepts.

Le troisième type d'activités - la discussion des textes étudiés - permet:

- de construire chez les apprenants l'habitude d'employer le phénomène grammatical étudié d'une manière consciente (ce n'est plus une simple mémorisation/ répétition/ reproduction des formes rencontrées dans les textes); 
- de rediscuter en groupe Tandem les cas difficiles (là où les apprenants continuent d'employer des formes incorrectes malgré les discussions préalables) - ainsi, c'est une sorte de rétroaction (feed-back) pour l'enseignant.

Nous essayons d'élaborer des exercices servant à assurer le transfert du savoir conceptuel au savoir opératoire. Ces exercices sont présentés sous forme de tâches qui demandent d'analyser les concepts gramaticaux exprimés par des moyens grammaticaux ou lexicaux dans des textes en langue étrangère et maternelle afin de trouver les convergences et les divergences de l'expression du même concept dans les deux langues.

Voici quelques exercices que nous proposons au cours de grammaire contrastive qui sont créés sur un extrait du roman de Anna Gavalda "Je l'aimais 》. Les étudiants russes sont divisés en sous-groupes. Chaque sous-groupe travaille avec un étudiant francophone. On étudie le thème "Concept grammatical de détermination : Article "

Tâche 1. Lisez le texte ci-dessous, mettez les articles convenables ou la préposition " de " là où il le faut. Essayez de justifier leur emploi. Révélez les noms déterminés par la situation, par le contexte, post-déterminés. Quels moyens linguistiques expriment la détermination / indétermination en russe:

- Qu'est-ce que je peux faire pour vous aider?

- Tiens, coupe-moi ça, a dit mon beau-père, en me tenant trois oignons.

J'ai attrapé __ planche en bois près de évier et je me suis assise en face de

lui. Son visage était de nouveau contracté. On entendait seulement bruits de feu.

172 - Vous avez meilleurs amis, vous?

- Pourquoi tu me demandes ça?

- Comme ça. Enfin... Je n'en sais rien. Je ne vous ai jamais entendu en parler.

Mon beau-père s'appliquait sur ses rondelles de amusant de regarder homme qui fait carottes. C'est toujours de sa vie. Cette façon de suivre recette à première fois Mathiot était déesse très susceptible.

- Il y a marqué « couper carottes en rondelles de taille moyenne ", tu crois que ça ira comme ça?

- C'est parfait!

- Merci... Où j'en étais déjà? Ah oui, mes amis... En fait, j'en ai eu trois... Patrick, que j'ai connu pendant voyage à Rome, Jean Théron, que tu connais, et mon frère, Paul, que tu n'as jamais vu puisqu'il est mort en 56...

Il faisait revenir oignons et carottes dans cocotte avec morceaux de poitrine fumée, ça sentait très bon.

- Vous considérez votre frère comme votre meilleur ami?

- Il était plus que ça encore... C'était garçon fin, drôle, attentif à uns et $\grave{a}$ autres. Il connaissait chant de tous oiseaux. C'était garçon

charmant. Vraiment charmant.

- De quoi est-il mort?

- Il est allé en Indochine. Il en est revenu malade et à moitié fou. Il est mort de tuberculose 14 juillet 1956

Tâche 2. Restituez les articles dans les phrases suivantes. Choisissez le remplaçant russe convenable pour chaque article. Traduisez ces phrases en russe :

- As-tu ........meilleurs amis (несколько/ какие-то/ какие-нибудь/любые)? 
- Prenez trois carottes. Coиреz ..... carottes (несколько/ какие-нибудь/ эти/ любые) en rondelles. Faites revenir ........carottes (несколько/ какие-нибудь/ эти/ любые) dans ......cocotte (одной/ какой-то/ любой/ этой/ какой-нибудь).

- Je n'ai pas regardé ..... seul film (ни одного из всех возможных, где он снимался) единственного, который существует) аvec cet acteur.

- Elle est ..... seule qui a survécu (одна из нескольких возможных/ единственная).

- Il y est allé à cause de ..... fille (одной/ какой-то/ какой-нибудь/ каждой/ любой). ....... histoire absudre (Одна/ эта/ какая-то/ какая-нибудь).

Tâche 3. Traduisez en français:

Утром Сергей идет на кухню, открывает холодильник, достает масло $и$ бутылку апельсинового сока. Потом он включает кофеварку, чтобы сварить кофе. Пока кофе варится, Сергей берет нож и делает себе три бутерброда. Ну вот, кофе готов. Сергей садится за стол, слушает радио, пьет кофе, ест бутерброды.

Tâche 4. Choisissez les remplaçants russes convenables pour les articles dans les phrases suivantes, là où il y en a. Justifiez votre choix :

- Il y a du dédain dans votre conduite (некоторое количество / какое-то).

- Je vivais dans un immeuble tout gris (единственно существующем / каком-то / каком-нибудь).

- Nous habitions dans un appartement sinistre où le soleil n'entrait jamais (единственно существующем / одном из / каком-нибудь)

- En dessous de notre appartement il y avait un dépôt. (какой-то/ один из/ единственный/ какой-нибудь)

- Je l'ai invitée à boire un café sous les arcades (какого-то / чашку).

- Tи пе sais pas quoi faire? Lis un livre! (какой-то/ какой-нибудь)

Tâche 5. Remplissez le tableau en indiquant quels remplaçants russes correspondent au trait sémantique de spécificité/ non-spécificité :

\begin{tabular}{|c|l|c|}
\hline Remplaçant & Spécificité & $\begin{array}{c}\text { Non- } \\
\text { spécificité }\end{array}$ \\
\hline единственно существующчй & & \\
\hline какой-то & & \\
\hline какой-нибудь (любой) & & \\
\hline одиниз & & \\
\hline
\end{tabular}

Tâche 6. Parmi les phrases en russe ci-dessous barrez celles qui vous paraissent incorrectes. En quoi consiste le conflit dans les phrases incorrectes ?Reliez les phrases correctes avec leurs équivalents en français. Dites, avec quels moyens peuton exprimer " une quantité limitée " en russe?

\begin{tabular}{|l|l|}
\hline En russe & En français \\
\hline - Я съел супа & $\begin{array}{l}\text { J'ai mangél fini ma soupe. } \\
\text { J'ai mangé de la soupe. } \\
\text { Je mange de la soupe } \\
\text { Je mange ma soupe. } \\
\text { - Я съел суn. }\end{array}$ \\
\hline - Я поел супа assez mangé de la \\
\hline
\end{tabular}




\begin{tabular}{|c|c|}
\hline - Я поел суп. & \multirow[t]{5}{*}{$\begin{array}{l}\text { soupel } \\
\text { J'en ai mangé à ma faim. }\end{array}$} \\
\hline - Я ем суп. & \\
\hline - Я ем супа. & \\
\hline - Я наелся суп. & \\
\hline - Я наелся супа. & \\
\hline
\end{tabular}

\section{Exemple d'un commentaire grammatical:}

Во французском языке частичность выражается артиклем du, de la (любой формой частичного артикля). В русском языке это значение может выражаться морфологически - противопоставлением родительного $и$ винительного падежей - только в функичи прямого дополнения.

В русском языке выражение количественной неопределенности может не ограничиваться одним именем, но также затрагивать связанный с ним глагол. Некоторые глагольные видовые префиксы (на-, пона-, на-..-ся) выражают количественную характеристику действия, степень охвата им объекта, в частности количественную неопределенность и множественность объектов. Глаголь с такими префиксами требуют обязательно родительного падежа, выражающего количественную неопределенность.

174 Ce type d'exercices amène les étudiants à analyser les nuances sémantiques exprimées par telle ou telle catégorie grammaticale, à travailler sur le conduite explicative, à mettre en jeu de nombreuses compétences. En outre, ce travail collaboratif nécessite la prise de responsabilité. Il est toujours difficile à un locuteur natif d'expliquer pourquoi il emploie telle ou telle forme grammaticale. Néanmoins, la prise de conscience des concepts grammaticaux dans la langue maternelle facilite la construction de la compétence grammaticale dans la langue étrangère. Sur le plan du développement cognitif, les activités de conscientisation grammaticale, poussant les étudiants à apprendre à généraliser et à systématiser dans le traitement de l'information, créent le contexte professionnel de la formation linguistique dans l'optique du traducteur et mettent en évidence les objectifs pratiques et formateurs à atteindre.

\section{L'interaction}

Ce type de cours est dédié au développement de la compétence interactionnelle des apprenants. L'interaction se fait autour d'un document déclencheur - un texte, une vidéo, une idée avancée par le professeur, l'exposé préparé par un des étudiants.

La présence d'un apprenant francophone enrichit énormément les cours mais le professeur doit savoir en profiter et la rendre utile pour tous les participants. Tandem s'avère un instrument quasi-idéal pour aider les étudiants à acquérir la compétence d'interaction interculturelle, ce qui est un vrai défi pour tous. Partant de l'idée qu'une vraie interaction interculturelle n'est possible que dans des situations d'échange réel entre les représentants de cultures différentes, les cours de langue se basent sur des situations authentiques à la croisée de deux cultures.

\section{Les activités}

Le support pédagogique utilisé pendant les cours est "Le français pour les futurs traducteurs / interprètes. Il n'est pas conçu spécialement pour les cours Tandem mais les principes sur lesquels nous nous sommes appuyés en l'élaborant le rendent assez 
efficace pour cette méthode aussi. Ces principes sont la professionnalisation de l'apprentissage de la langue, l'attention équilibrée envers la langue étrangère et celle maternelle, la contextualisation de l'apprentissage. Les unités étudiées sont "La nourriture et la cuisine", "La santé et la médecine", "Le commerce et la consommation", "Les vacances et les voyages", c'est-à-dire l'apprentissage se fait autour des sujets quotidiens. Chaque unité commence par la présentation d'une situation dans laquelle on aura besoin d'un médiateur. Le groupe est invité à analyser la situation proposée, à faire son praxéogramme (la succession d'actions) en la décomposant en étapes, à citer les unités lexicales et les collocations thématiques dont on aura besoin. Tout cela est fait pour les deux cultures et langues.

Chaque activité proposée par le professeur doit être analysée du point de vue de son utilité professionnelle. Le lien entre l'activité d'apprentissage et celle professionnelle peut être assez court (Vous allez assurer la communication de votre ami français qui ne parle pas russe avec le médecin d'une clinique russe) mais peut ne pas être suffisamment visible (Présentez à votre ami français le système médical russe). Chaque fois le professeur s'assure que les étudiants comprennent dans quel but on fait telle ou telle activité, quelles stratégies développe-t-elle. Prenons l'exemple d'une situation :

«Un collègue étranger vous propose de déjeuner ensemble dans un restaurant. Vous acceptez la proposition mais vous le prévenez que vous vous y rendrez avec votre mère qui est en visite chez vous et vous ne voulez pas la laisser seule. Votre mère ne parle aucune langue étrangère. Vous allez assurer la communication lors de ce déjeuner et décidez de vous préparer un peu. Pour cela vous devez répondre aux questions suivantes :

1. De quelle information socioculturelle vous devez tenir compte?

2. De quelles étapes se compose un déjeuner au restaurant? (entrer, saluer, présenter,...)

3. De quelles unités lexicales et collocations vous aurez besoin?"

Le professeur divise le groupe en sous-groupes dont l'effectif est monoculturel. Chaque sous-groupe répond à ces questions en imaginant que la situation va se dérouler dans son pays d'origine. Après quelques minutes de réflexion les sousgroupes présentent leurs réponses en leur langue maternelle. Les autres sous-groupes sont invités à ajouter des précisions, à poser des questions. La discussion se fait en langue de la présentation.

Il est à noter que le recours à la langue maternelle à cette étape est expliqué par les particularités de notre cognition. L'acquisition d'une nouvelle connaissance est plus efficace si on la base sur les connaissances déjà existantes. Cette activité est donc centrée sur l'activation des connaissances, leur mobilisation, dans la culture maternelle et leur systématisation. De plus, l'utilisation de la langue maternelle ne limite pas la production, on n'a pas de risque qu'une idée soit rejetée faute de vocabulaire. Celui-ci sera actualisé lors de l'analyse collective de la présentation des sous-groupes étrangers où il faudra demander des précisions, des explications.

L'étape de la présentation est très importante et demande une grande implication de tous les participants. Ceux qui présentent leurs réponses doivent les adapter aux interlocuteurs étrangers. L'adaptation dans ce cas n'est pas synonyme de la simplification. Ce discours explicatif dans le Tandem a un objectif supplémentaire - le présentateur apprend sa langue maternelle à l'autre. Et là il s'avère que expliquer quelque chose à un étranger même en sa langue maternelle est une tâche assez difficile. Les stratégies de l'explication, la reformulation, la « traduction intralinguale ", la « traduction intersémiotique » obtiennent une nouvelle valeur pratique en cours de langue et du coup un nouvel élan du développement. 
Tous les étudiants, russophones aussi bien que francophones, avouent que grâce à des situations pareilles ils se sont construit une nouvelle image de leur langue maternelle. Ils ont commencé à la considérer comme un outil professionnel indispensable - l'idée qu'on tâche de suggérer dès le début de leur formation mais à laquelle ils ne se sensibilisent pas faute de cette expérience.

A l'étape de l'analyse des réponses, les étudiants, guidés par le professeurs, apprennent à découvrir les ressemblances et différences au niveau culturel, comportemental, langagier et essayent de trouver des moyens pour approcher les représentants des deux cultures tout en respectant leurs particularités.

La dernière étape prévoit un jeu de rôle simulant la situation décrite en tenant compte des résultats de l'analyse.

Plongés dès le début dans l'environnement biculturel les étudiants apprennent à analyser le langage du locuteur natif, à adopter l'attitude qui convient à la situation.

De temps en temps on invite à ces cours les russes qui ne parlent pas français et organise des discussions aux sujets différents.

Les stratégies et les compétences acquises grâce à l'apprentissage en Tandem stimulent la participation active des apprenants aux événements interculturels organisés à la faculté et en ville. Ces activités interculturelles engagent nos étudiants dans les pratiques discursives interactionnelles. Ce sont, par exemple, «Fête de la gastronomie », « Fête des langues », « Nuit à la bibliothèque » etc.

\section{En guise de conclusion}

En réalisant l'approche actionnelle combinant les éléments des approches communicative, active, cognitive, interculturelle et contextuelle l'enseignement en Tandem donne lieu au partage de connaissances et d'expériences vécues, de stratégies communicatives interculturelles, de visions différentes du monde et la prise de conscience des concepts de base de la culture maternelle. Dans notre vision, la méthode de Tandem devient efficace si elle se base sur les principes essentiels suivants:

- $\quad$ l'apprentissage collaboratif se réalise dans le contexte professionnalisé ;

- articulation autour des concepts et valeurs de base dans les deux langues et cultures ;

- focalisation sur les concepts grammaticaux, sur la grammaire contrastive et non sur les règles de grammaire ;

- focalisation pragmatique sur le bagage cognitif, thésaurus thématique et non sur les listes de lexique ;

- $\quad$ analyse comparative et contrastive des concepts culturels et professionnels ;

- appui sur l'expérience communicative, voire professionnelle, vécue des apprenants.

\section{Bibliographic references}

ABDALLAH-PRETCEILLE, M. 1995. L'education interculturelle en France du devant de la scene aux coulisses, in Identites et cultures à l'ecole, Migrants-Formation, $\mathrm{n}^{\circ} 102$, septembre.

ABRY, D. 2007. Les activites pour la classe et le CECR, Conference proferee dans le Colloque International de la FIPF : Le Cadre Europeen, une reference mondiale ? Sevres, de 19 à 21 juin 2007.

CECR. Conseil de l'Europe, Conseil de la cooperation culturelle, comite de l'education, division des langues vivantes. Cadre europeen commun de reference pour les langues : apprendre, enseigner, evaluer. Paris : Didier, 2001. Disponible en version pdf sur : http://www.coe.int/t/dg4/linguistic/Source/Framework_fr.pdf 
CUQ J-P. - GRUCA I. 2003. Cours de didactique du francais langue etrangere. Grenoble: Presses Universitaires de Grenoble. -452 p.

DAHOUT, J-C. - QUENIART, C. 2000. « De quoi un traducteur diplome doit-il etre capable ? ", In: Gouadec, Daniel (ed). Formation des traducteurs. Actes du colloque international de Rennes 2 (24-25 septembre 1999) Paris: La maison du dictionnaire. Publie en ligne : www.christianpuren.com/mestravaux-liste-et-liens/2002b/

GALISSON, R. 1986. Eloge de la didactologie/didactique des langues et des cultures. Etudes de Linguistique Appliquee $\mathrm{N}^{\circ} 64$. Paris : Didier Erudition, pp. 39-54.

GOUADEC, D. 2002. Profession : traducteur. Paris: La maison du dictionnaire. ISBN 2856081738

GOUADEC D. 2003 Professionnaliser In: Traduction, terminologie, redaction. Actes des universites d'ete et d'automne 2002 et du colloque international: Specialites et specialisations dans la pratique professionnelle et la formation des traducteurs. Universite de Rennes 2. Paris: La maison du dictionnaire. pp. 217-245.

LEWIS, T. - STICKLER, U. 2007. Les stratégies collaboratives d'apprentissage lors d'un échange en tandem via Internet, Lidil, 36. 2007, pp. 163-188

PUREN, Ch. 2002. "Perspectives actionnelles et perspectives culturelles en didactique des langues-cultures : vers une perspective co-actionnelle co-culturelle », In: Les Langues modernes n ${ }^{\circ} 3 / 2002$, juil.-août-sept., Paris: APLV. pp. 55-71.

PORSHNEVA, E. - KRASNOVA, M. 2012. " Initiation à l'apprentissage d'une langue etrangere dans l'optique du traducteur: creation du contexte professionnel », In: Didactique du FLE dans les pays slaves: revue scientifique internationale. Edition SAUF V.3, Numero 2, pp. 12-19 Nitra, Slovaquie. ISSN 1337-9283,

PORSHNEVA, E. - SPIRIDONOVA, O. 2008. " La conscientisation grammaticale dans le contexte des etudes specialisees du FLE » dans La langue francaise et la communication interculturelle, Actes $d u$ colloque international 25-26 mars, Pyatigorsk, Universite Linguistique. P.228-235

PORSHNEVA, E.R. 2004. Mezhdistsiplinarnyie osnovyi bazovoy lingvisticheskoy podgotovki spetsialista-perevodchika: Avtoref.dis.na soisk.uchen.step.d-r ped.nauk: (13.00.08) / E. R. Porshneva; Nizhegorod.gos.lingv.un-t im.N.A.Dobrolyubova. N. Novgorod. Kazan

RICHTERICH, R. 1985. Besoins langagiers et objectifs d'apprentissage, recherches et applications. Paris: Hachette. ISSN 0768-360X

VERBITSKY A.A. 1991. Formation active à l'ecole superieure: approche contextuelle. Moscou : Ecole superieure.

VYGOTSKY L. S. 1978. Mind in Society [Le mental dans la societe], Cambridge, Mass., Harvard University Press.

prof. Elena Porshneva

dr. Indira Abdulmianova

Department of french studies, translation and interpreting

University State Linguistic University N.A. Dobrolyubov Nizhny Novgorod

Address 31a Minina street

Nizhny Novgorod

State Russia

eporshneva@gmail.com

i.r.abdulmyanova@lunn.ru 